Pacific Journal of Mathematics

GLOBALIZATION THEOREMS FOR LOCALLY FINITELY 


\section{GLOBALIZATION THEOREMS FOR LOCALLY FINITELY GENERATED MODULES}

\section{ROGER WIEGAND}

Each commutative ring has a coreflection $\hat{R}$ in the category of commutative regular rings. We use the basic properties of $\hat{R}$ to obtain globalization theorems for finite generation and for projectivity of $R$-modules.

1. Preliminaries. A detailed description of the ring $\hat{R}$ may be found in [8]. Here we list without proofs the facts that will be needed. We assume that everything is unitary, but not necessarily commutative. However, $R$ will always denote an arbitrary commutative ring. All unspecified tensor products are taken over $R$. For each $a \in R$ and each $P \in \operatorname{Spec}(R)$, let $a(P)$ be the image of $a$ under the obvious map $R \rightarrow R_{P} / P R_{P}$. Then $\hat{R}$ is the subring $\amalg_{P} R_{P} / P R_{P}$ consisting of finite sums of elements $[a, b]$, where $[a, b]$ is the element whose $P^{\text {th }}$ coordinate is 0 if $b \in P$ and $a(P) / b(P)$ if $b \notin P$. There is a natural homomorphism $\varphi: R \rightarrow \hat{R}$ taking $a$ to $[a, 1]$. The ring $\hat{R}$ is regular (in the sense of von Neumann). The statement that $\hat{R}$ is a coreflection means simply that each homomorphism from $R$ into a commutative regular ring factors uniquely through $\varphi$.

The map $\operatorname{Spec}(\varphi): \operatorname{Spec}(\hat{R}) \rightarrow \operatorname{Spec}(R)$ is one-to-one and onto; for each $P \in \operatorname{Spec}(R)$ we let $\hat{P}$ be the corresponding prime (= maximal) ideal of $\hat{R}$.

If $A$ is an $R$-module and $P \in \operatorname{Spec}(R)$, then $A_{P} / P A_{P}$ and $(A \otimes \hat{R})_{\hat{P}}$ are vector spaces over $R_{P} / P R_{P}$ and $\hat{R}_{\hat{P}}$ respectively. The map $\varphi: R \rightarrow \hat{R}$ induces an isomorphism $R_{P} / P R_{P} \cong \hat{R}_{\hat{P}}$, and, under the identification, $A_{P} / P A_{P}$ and $(A \otimes \hat{R})_{\hat{P}}$ are isomorphic vector spaces.

\section{Globalization theorems.}

LEMmA. If $A \otimes \hat{R}=0$ and $A_{R}$ is locally finitely generated then $A=0$.

Proof. For each prime $P, A_{P} / P A_{P}=0$, by the last paragraph of $\S 1$. Since $A_{P}$ is finitely generated over $R_{P}$, Nakayama's lemma implies that $A_{P}=0$ for each $P \in \operatorname{Spec}(R)$. Therefore $A=0$.

THEOREM 1. Assume $(A \otimes \hat{R})$ is finitely generated over $\hat{R}$, and that $A_{R}$ is either locally free or locally finitely generated. Then $A_{R}$ is finitely generated. 
Proof. Assume $A_{R}$ is locally free. Then, for each prime $P, A_{P}$ is a direct sum of, say, $\kappa$ copies of $R_{P}$. Then $A_{P} / P A_{P}$ is a direct sum of $\kappa$ copies of $R_{P} / P R_{P}$. But since $(A \otimes \hat{R})$ is finitely generated over $\hat{R}, A_{P} / P A_{P}$ is finite dimensional over $R_{P} / P R_{P}$. Thus $\kappa$ is finite, and we conclude that $A_{R}$ is locally finitely generated.

Now, if $A_{R}$ is not finitely generated, we can express $A$ as a wellordered union of submodules $A_{\alpha}$, each of which requires fewer generators than $A$. We will get a contradiction by showing that some $A_{\alpha}=A$. Let $B_{\alpha}=\operatorname{Im}\left(A_{\alpha} \otimes \hat{R} \rightarrow A \otimes \hat{R}\right)$. Since

$$
A \otimes \hat{R}=\lim _{\vec{\alpha}}\left(A_{\alpha} \otimes \hat{R}\right), \quad A \otimes \hat{R}=\bigcup_{a} B_{\alpha}
$$

Since the $B_{\alpha}$ are nested and $(A \otimes \hat{R})$ is finitely generated over $\hat{R}$, some $B_{\alpha_{0}}=A \otimes \hat{R}$, that is, $A_{\alpha_{0}} \otimes \hat{R} \rightarrow A \otimes \hat{R}$. Let $C=A / A_{\alpha_{0}}$. Then $C \otimes \hat{R}=\operatorname{Coker}\left(A_{\alpha_{0}} \otimes \hat{R} \rightarrow A \otimes \hat{R}\right)=0$, and $C_{R}$ is certainly locally finitely generated. By the lemma, $C=0$, and $A_{\alpha_{0}}=A$.

THEOREM 2. Let $A_{R}$ be finitely generated and flat, and assume $(A \otimes \hat{R})$ is $\hat{R}$-projective. Then $A_{R}$ is projective.

Proof. By Chase's theorem [3, Theorem 4.1] it is sufficient to show that $A_{R}$ is finitely related. Let $0 \rightarrow K \rightarrow F \rightarrow A \rightarrow 0$ be an exact sequence, with $F_{R}$ free of finite rank. This sequence splits locally, so $K$ is locally finitely generated. Since $A_{R}$ is flat, the long exact sequence of Tor shows that $0 \rightarrow K \otimes \hat{R} \rightarrow F \otimes \hat{R} \rightarrow A \otimes \hat{R} \rightarrow 0$ is exact. This sequence splits, so $(K \otimes \hat{R})$ is finitely generated over $\hat{R}$. By Theorem $1, K_{R}$ is finitely generated.

3. Applications. The following result generalizes the wellknown fact that over a noetherian ring every finitely generated flat module is projective.

Proposition 1. If $R$ has a.c.c. on intersections of prime ideals then every finitely generated flat $R$-module is projective.

Proof. In [8] these rings are characterized as those for which $(A \otimes \hat{R})$ is $\hat{R}$-projective for every finitely generated $A_{R}$. The conclusion follows from Theorem 2.

Suppose $A_{R}$ is locally finitely generated. For each prime ideal $P$ let $r_{A}(P)$ denote the number of generators required for $A_{P}$ over $R_{P}$. By Nakayama's lemma, $r_{A}(P)=d_{A}(\hat{P})$, the dimension of $(A \otimes \hat{R})_{\hat{P}}$ as a vector space over $\hat{R}_{\hat{P}}$. Since the map $\hat{P} \rightarrow P$ is continuous, it follows that if $r_{A}$ is continuous on $\operatorname{Spec}(R)$ then $d_{A}$ is continuous on Spec $(\hat{R})$. Using these observations we can give easy proofs of the 
following two theorems:

Theorem 3 (Bourbaki [1, Th. 1]): Assume $A_{R}$ is finitely generated and flat, and that $r_{A}$ is continuous. Then $A_{R}$ is projective.

Theorem 4 (Vasconcelos [7, Prop. 1.4]): Assume $A_{R}$ is projective and locally finitely generated, and that $r_{A}$ is continuous. Then $A_{R}$ is finitely generated.

Proof of Theorem 3. By Theorem 3 we may assume $R$ is regular. A proof of Theorem 3 in this case may be found in [5], but we include one here for completeness. For each $k \geqq 0$ let

$$
U_{k}=\left\{P \in \operatorname{Spec}(R) \mid r_{A}(P)=k\right\} \text {. }
$$

By hypothesis the sets $U_{k}$ are clopen, and we let $e_{k}$ be the idempotent with support $U_{k}$. Then $A=A e_{0} \oplus \cdots \oplus A e_{n}$, and $r_{A e_{k}}$ is constant on $\operatorname{Spec}\left(R e_{k}\right)$. Therefore we may assume $r_{A}$ is constant on $\operatorname{Spec}(R)$, say $r_{A}(P)=n$ for all $P$. Given a prime $P$, choose $a_{1}, \cdots, a_{n} \in R$ such that $a_{1}(P), \cdots, a_{n}(P)$ span $A_{P}$. Then $a_{1}(Q), \cdots, a_{n}(Q)$ span $R_{Q}$ for all $Q$ in some neighborhood of $P$. (Here we need $A_{R}$ finitely generated.) In this way we get a partition of $\operatorname{Spec}(R)$ into disjoint clopen sets $V_{1}, \cdots, V_{m}$ together with elements $a_{i j} \in R$ such that $a_{i j}(P), \cdots, a_{n j}(P)$ span $A_{P}$ for each $P \in V_{j}$. Let $e_{j}$ be the idempotent with support $V_{j}$, and set $b_{i}=\Sigma_{j} e_{j} a_{i j}$. Then, if $P_{R}$ is free on $u_{1}, \cdots, u_{n}$, the map $P \rightarrow A$ taking $u_{i}$ to $b_{i}$ is an isomorphism locally, and therefore globally.

Proof of Theorem 4. By Theorem 1 and the proof of Theorem 3 we can assume $R$ is regular and $r_{A}(P)=n$ for all $P$. Write $A=$ $\bigoplus \sum_{i \in I} R e_{i}, e_{i}^{2}=e_{i} \neq 0$, by [4]. Given $P \in \operatorname{Spec}(R)$, since $\left(R e_{i}\right)_{P}$ is 0 if $e_{i} \in P$ and $R_{P}$ if $e_{i} \notin P$, we see that there are precisely $n$ indices $i$ for which $e_{i} \notin P$. For each $n$-element subset $J \subseteq I$ let

$$
U(J)=\left\{P \in \operatorname{Spec}(R) \mid e_{j} \notin P \text { for each } j \in J\right\} .
$$

These open sets cover $\operatorname{Spec}(R)$, so $\operatorname{Spec}(R)=U\left(J_{1}\right) \cup \cdots \cup U\left(J_{m}\right)$. If $j \notin J_{1} \cup \cdots \cup J_{m}$ then $e_{j}$ is in every prime ideal, contradicting $e_{j} \neq 0$. Therefore $|I| \leqq m n$, and $A_{R}$ is finitely generated.

As a final application we give the following:

Proposition 2. Let $0 \rightarrow A \rightarrow B \rightarrow C \rightarrow 0$ be an exact sequence of flat $R$-modules Assume $A_{R}$ is finitely generated and $(B \otimes \hat{R})_{\hat{R}}$ is projective. Then $A_{R}$ is projective.

Proof. Since $C_{R}$ is flat, $0 \rightarrow A \otimes \hat{R} \rightarrow B \otimes \hat{R} \rightarrow C \otimes \hat{R} \rightarrow 0 \quad$ is 
exact. Since $\hat{R}$ is semihereditary $(A \otimes \hat{R})$ is $R$-projective. By Theorem $2, A_{R}$ is projective.

If $B_{R}$ is projective this proposition contains no new information. (In fact, a trivial extension of Chase's Theorem shows that the sequence splits.) On the other hand, if we let $M_{R}$ be projective, take $f \in R$, and let $B=M_{f}=\left\{\left[m / f^{n}\right]\right\}$, then $B_{R}$ is not in general projective; but by the second corollary to Theorem 5 (next section), $B \otimes \hat{R}$ is $\hat{R}$-projective.

4. Epimorphisms. Suppose $M$ is a multiplicative subset of $R$, and let $S=M^{-1} R$. Since $S \otimes \hat{R}_{\hat{P}}=S_{P} / P S_{P}$ for each prime $P$, we see that $S \otimes \hat{R}_{\hat{P}}$ is $\hat{R}_{\hat{P}}$ if $P \cap M=\varnothing$, and 0 if $P \cap M \neq \varnothing$. If we could show that $(S \otimes \hat{R})_{\hat{R}}$ is finitely generated, it would follow easily that $S \otimes \hat{R}=\hat{R} / K$, where $K$ is the intersection of those primes $\hat{P}$ for which $P \cap M=\varnothing$. We give an indirect proof of this fact in a more general setting.

Suppose $R$ and $S$ are commutative rings and that $\alpha: R \rightarrow S$ is an epimorphism in the category of rings. By a theorem of Silver [6] this is equivalent to the natural map $S \otimes S \rightarrow S$ being an isomorphism. It is known [8] that $R \rightarrow \hat{R}$ is an epimorphism, and it follows readily that the natural maps $f: S \rightarrow S \otimes \hat{R}$ and $g: R \rightarrow S \otimes \hat{R}$ are epimorphisms.

THEOREM 5. Let $R$ and $S$ be commutative rings and let $\alpha: R \rightarrow S$ be an epimorphism in the category of rings. Then there is a unique ring homomorphism $\beta: \widehat{S} \rightarrow S \otimes \widehat{R}$ making the following diagram commute:

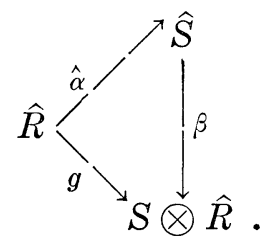

Moreover, $\beta$ is an isomorphism, and $\hat{\alpha}$ and $g$ are surjections with kernel $K=\cap\left\{\hat{P} \mid S_{P} \neq P S_{P}\right\}$.

Proof. We first show that $S \otimes \hat{R}$ is regular. Suppose $A$ and $B$ are $(S \otimes \hat{R})$-modules. Then by Silver's Theorem $B=S \otimes_{R} B$, and by $[2$, p. 165] we have

$$
A \otimes_{S \otimes \hat{R}} B=A \otimes{ }_{S \otimes \hat{R}}\left(S \otimes_{R} B\right)=\left(A \otimes_{S} S\right) \otimes_{\hat{R} \otimes R} B=A \otimes_{\hat{R}} B .
$$

It follows that tensor products over $S \otimes \hat{R}$ are exact, and therefore 
$S \otimes \hat{R}$ is regular. Hence there is a unique map $\beta: \hat{S} \rightarrow S \otimes \hat{R}$ such that $\beta \varphi_{S}=f$, where $\varphi_{S}: S \rightarrow \hat{S}$ is the natural map. Consider the diagram:



Here $\gamma$ is defined by the equations $\gamma f=\varphi_{S}, \gamma g=\hat{\alpha}$. Now $\gamma \beta \varphi_{S}=$ $\gamma f=\varphi_{S}$ and $\beta \gamma f=\beta \varphi_{S}=f$. Since $\varphi_{S}$ and $f$ are both epimorphisms, we see that $\gamma=\beta^{-1}$. Also, $B \widehat{\alpha}=B \gamma g=g$, as required. Uniqueness of $\beta$ follows from the fact that $\hat{\alpha}$ is an epimorphism (since both $\alpha$ and $\varphi_{S}$ are).

Next, we show $\hat{\alpha}$ is onto. To simplify notation, we assume $R$ is regular and $\alpha: R \rightarrow S$ is an epimorphism. Then $S \otimes S \stackrel{\mu}{\rightarrow} S$ is an isomorphism. But then $S_{P} \otimes_{R_{P}} S_{P} \rightarrow S_{P}$ is an isomorphism for each $P \in \operatorname{Spec}(R)$. If $s \in S_{P}$ then $1 \otimes s-s \otimes 1 \in \operatorname{ker} \quad \mu_{P}=0$. It follows that the dimension of $S_{P}$ as a vector space over $R_{P}$ is either 0 or 1 . Therefore $\alpha_{P}$ is surjective for each $P,(\alpha(1)=1)$, and we conclude that $\alpha$ is surjective.

Finally, we compute $\operatorname{ker} g=K$. If $P \in \operatorname{Spec}(\hat{R})$, then

$$
K \cong \hat{P} \Longleftrightarrow K_{\hat{P}}=0 \Longleftrightarrow \hat{S}_{\hat{P}} \neq 0 \Longleftrightarrow S \otimes \hat{R}_{\hat{P}} \neq 0 \Longleftrightarrow S_{P} / P S_{P} \neq 0 .
$$

CoRollaRy 1. Let $M$ be a multiplicative subset of $R$ and let $S=M^{-1} R$. Then $S \otimes \hat{R}$ is a cyclic $\hat{R}$-module, and $S \otimes \hat{R}$ is $\hat{R}$ projective if and only if $\{\hat{P} \mid M \cap P \neq \varnothing\}$ is closed in $\operatorname{Spec}(\hat{R})$.

Proof. Let $K$ be as in Theorem 5. Then $S \otimes \hat{R}=\hat{R} / K$ is $\hat{R}$ projective if and only if $K$ is a principal ideal, that is, if and only if the set of primes containing $K$ is open in $\operatorname{Spec}(\hat{R})$. But

$$
\hat{P} \supseteqq K \Longleftrightarrow P S_{P} \neq S_{P} \Longleftrightarrow M \cap P=\varnothing \text {. }
$$

The next corollary shows that Theorem 2 is false if $A_{R}$ is not assumed to be finitely generated.

Corollary 2. For each $f \in R, R_{f} \otimes \hat{R}$ is $\hat{R}$-projective. 
Proof. Set $M=\left\{f^{n}: n \geqq 0\right\}$. Then $P \cap M \neq \varnothing$ if and only if $\varphi(f) \in \hat{P}$. Thus $K$ is the principal ideal of $\hat{R}$ generated by $\varphi(f)$, and $\hat{R} / K$ is $\hat{R}$-projective.

\section{REFERENCES}

1. N. Bourbaki, Eléments de mathématique. Algèbre commutative, Chapter II, Hermann, Paris, 1961.

2. H. Cartan and S. Eilenberg, Homological algebra, Princeton Univ. Press, Princeton, 1956.

3. S. U. Chase, "Direct products of modules", Trans. Amer. Math. Soc., 97 (1960), 457-473.

4. I. Kaplansky, Projective modules, Ann. of Math., 68 (1958), 372-377.

5. R. S. Pierce, Modules over commutative regular rings, Memoirs Amer. Math. Soc., 70 (1967).

6. L. Silver, Non-commutative localizations and applications, J. Algebra, 7 (1967), 4476.

7. W. Vasconcelos, On projective modules of finite rank, Proc. Amer. Math. Soc., 22, No. 2 (1969), 430-433.

8. R. Wiegand, Modules over universal regular rings, (to appear in the Pacific J. Math.).

Received March 19, 1971. This research was partially supported by NSF Grant GP-19102.

UNIVERSITY OF WISCONSIN 


\section{PACIFIC JOURNAL OF MATHEMATICS}

\section{EDITORS}

H. SAMELSON

Stanford University

Stanford, California 94305

C. R. HobBY

University of Washington

Seattle, Washington 98105
J. DugundjI

Department of Mathematics

University of Southern California

Los Angeles, California 90007

RICHARD ARENS

University of California

Los Angeles, California 90024

\section{ASSOCIATE EDITORS}

E. F. BECKENBACH

B. H. NeUMaNN

F. WOLF

K. YoshidA

\section{SUPPORTING INSTITUTIONS}

UNIVERSITY OF BRITISH COLUMBIA

CALIFORNIA INSTITUTE OF TECHNOLOGY

UNIVERSITY OF CALIFORNIA

MONTANA STATE UNIVERSITY

UNIVERSITY OF NEVADA

NEW MEXICO STATE UNIVERSITY

OREGON STATE UNIVERSITY

UNIVERSITY OF OREGON

OSAKA UNIVERSITY
UNIVERSITY OF SOUTHERN CALIFORNIA STANFORD UNIVERSITY

UNIVERSITY OF TOKYO

UNIVERSITY OF UTAH

WASHINGTON STATE UNIVERSITY UNIVERSITY OF WASHINGTON

$\stackrel{*}{*} \stackrel{*}{*} \stackrel{*}{*}$ AMERICAN MATHEMATICAL SOCIETY
NAVAL WEAPONS CENTER 


\section{Pacific Journal of Mathematics}

Vol. 39 , No. 1

May, 1971

Charles A. Akemann, A Gelfand representation theory for $C^{*}$-algebras ....

Sorrell Berman, Spectral theory for a first-order symmetric system of

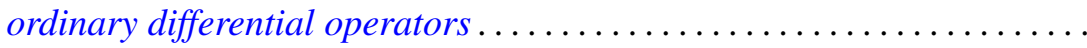

Robert L. Bernhardt, III, On splitting in hereditary torsion theories ........

J. L. Brenner, Geršgorin theorems, regularity theorems, and bounds for determinants of partitioned matrices. II. Some determinantal identities ..........................................

Robert Morgan Brooks, On representing $F^{*}$-algebras .............. 51

Lawrence Gerald Brown, Extensions of topological groups........... 71

Arnold Barry Calica, Reversible homeomorphisms of the real line ........ 79

J. T. Chambers and Shinnosuke Oharu, Semi-groups of local Lipschitzians in

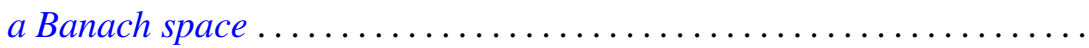

Thomas J. Cheatham, Finite dimensional torsion free rings .............

Byron C. Drachman and David Paul Kraines, A duality between

transpotence elements and Massey products ...................

Richard D. Duncan, Integral representation of excessive functions of a

Markov process ......................................

George A. Elliott, An extension of some results of Takesaki in the reduction



Peter C. Fishburn and Joel Spencer, Directed graphs as unions of partial



Howard Edwin Gorman, Zero divisors in differential rings ...

Maurice Heins, A note on the Löwner differential equations...

Louis Melvin Herman, Semi-orthogonality in Rickart rings. .

David Jacobson and Kenneth S. Williams, On the solution of linear G.C.D.

equations

Michael Joseph Kallaher, On rank 3 projective planes ... . .

Donald Paul Minassian, On solvable $O^{*}$-groups ...........

Nils Øvrelid, Generators of the maximal ideals of $A(\bar{D})$

Mohan S. Putcha and Julian Weissglass, A semilattice decomposition into

semigroups having at most one idempotent ............

Robert Raphael, Rings of quotients and $\pi$-regularity ....

J. A. Siddiqi, Infinite matrices summing every almost periodic sequence. .

Raymond Earl Smithson, Uniform convergence for multifunctions ...

Thomas Paul Whaley, Mulitplicity type and congruence relations in

universal algebras...

Roger Allen Wiegand, Globalization theorems for locally finitely generated modules... 